\title{
Precise Large Deviations of Aggregate Claims with Dominated Variation in Dependent Multi-Risk Models
}

\author{
Shijie Wang, ${ }^{1}$ Xuejun Wang, ${ }^{1}$ and Wensheng Wang ${ }^{2}$ \\ ${ }^{1}$ School of Mathematic Sciences, Anhui University, Hefei, Anhui 230601, China \\ ${ }^{2}$ Department of Mathematics, Hangzhou Normal University, Hangzhou 310036, China \\ Correspondence should be addressed to Shijie Wang; ahuwsj@126.com
}

Received 16 January 2014; Accepted 3 April 2014; Published 28 April 2014

Academic Editor: Beong In Yun

Copyright (c) 2014 Shijie Wang et al. This is an open access article distributed under the Creative Commons Attribution License, which permits unrestricted use, distribution, and reproduction in any medium, provided the original work is properly cited.

\begin{abstract}
We consider a dependent multirisk model in insurance, where all the claims constitute a linearly extended negatively orthant dependent (LENOD) random array, and then upper and lower bounds for precise large deviations of nonrandom and random sums of random variables with dominated variation are investigated. The obtained results extend some related existing ones.
\end{abstract}

\section{Introduction}

In a classic insurance risk model the surplus is described as the initial surplus plus the premium income with the claims taken off. Since asymptotic behavior for precise large deviations of the loss process of insurance risk models has theoretical significance and extensive applications, it has been widely investigated and there appeared to be a great deal of research literature. Some earlier works on precise large deviations can be found in Nagaev $[1,2]$, Heyde [3, 4], and Nagaev $[5,6]$, among others. Recent works on this field can be found in Cline and Hsing [7], Klüppelberg and Mikosch [8], Tang et al. [9], Tang [10], Liu [11], Chen and Zhang [12], Shen and Lin [13], Liu [14], Chen et al. [15], and Chen and Yuen [16], among others.

In consideration of insurance reality, some researchers have begun to focus on the precise large deviations of multirisk models in the past few years. See S. Wang and W. Wang [17], Lu [18, 19], He at al. [20], and S. J. Wang and W. S. Wang [21], among others. For convenience of representation, we adopt the notations of S. Wang and W. Wang [17]. Assume that the insurer manages $k$ types of insurance contracts at the same time, where $k$ is any fixed positive integer. The $i$ th related loss amounts (claims) are denoted by random variables $\left\{X_{i j}, j \geq\right.$ $1\}$ with common distribution $F_{i}(x)=P\left(X_{i} \leq x\right):=1-\bar{F}_{i}(x)$ satisfying $\bar{F}_{i}(x)>0$ for all $x \in(-\infty, \infty)$. Let $\left\{N_{i}(t), i=\right.$ $1, \ldots, k, t>0\}$ be independent nonnegative integer-valued counting processes independent of $\left\{X_{i j}, i=1, \ldots, k, j \geq 1\right\}$, satisfying $E N_{i}(t)=\lambda_{i}(t) \rightarrow \infty$ as $t \rightarrow \infty$. In insurance multi-risk models, $\left\{N_{i}(t), t>0\right\}_{i=1}^{k}$ always denote the $i$ th claim numbers of the related insurance contracts. Obviously, all the claims constitute the following random array:

$$
\left(\begin{array}{cccc}
X_{11} & X_{12} & X_{13} & \cdots \\
X_{21} & X_{22} & X_{23} & \cdots \\
\cdots & \cdots & \cdots & \cdots \\
X_{k 1} & X_{k 2} & X_{k 3} & \cdots
\end{array}\right) .
$$

Therefore, the loss process of the insurer during the period $[0, t]$ can be described as $S(k ; t)=\sum_{i=1}^{k} \sum_{j=1}^{N_{i}(t)} X_{i j}$. If the random arrays (1) are independent (i.e., to say all random variables $\left\{X_{i j}, i=1, \ldots, k, j \geq 1\right\}$ are independent) and all the claims have consistently varying tails (see the definition below), under some mild conditions, S. Wang and W. Wang [17] obtained the following result. For any fixed $\gamma>0$, as $t \rightarrow \infty$, the relation

$$
P\left(S(k ; t)-\sum_{i=1}^{k} \mu_{i} \lambda_{i}(t)>x\right) \sim \sum_{i=1}^{k} \lambda_{i}(t) \bar{F}_{i}(x)
$$

holds uniformly for $x \geq \max \left\{\gamma \lambda_{i}(t), i=1, \ldots, k\right\}:=\Gamma(k)$.

The relation (2) describes the so-called precise large deviations for random sums in multi-risk models. Subsequently, $\mathrm{Lu}[18,19]$ investigated precise large deviations with subexponential and long-tailed claims in multi-risk models under 
independent structures, respectively. He et al. [20] obtained the lower bounds of precise large deviations of multi-risk models with nonnegative random variables (regardless of heavy or light tails) under a specific dependence structure. However, on the one hand, for practical reasons, the independence assumptions in the above-mentioned papers are quite unrealistic. On the other hand, up to now, most works on precise large deviations with heavy tails concentrated on the consistent variation or some other heavy-tailed subclasses although some specific dependence structures have been considered. Therefore, it is more interesting to study the estimation of precise large deviation probabilities of aggregate claims in the presence of dominated variation and some dependence structures, where dominated variation strictly includes consistent variation. More recently, some researchers have begun to focus on this issue and obtained some interesting results. See Wang et al. [22], Wang et al. [23], Yang et al. [24], Yang and Wang [25], and Chen and Qu [26], among others. Motivated by the two reasons mentioned above, in this paper, upper and lower bounds for precise large deviations of aggregate claims with dominated variation in dependent multi-risk models (see the dependent structures in Definitions 1 and 2 below) are investigated. The obtained results extend some related existing ones.

To close this section, we introduce two new dependence structures called ENOD and LENOD, respectively, which are the basic assumptions in this paper. The idea of Definition 1 comes from Liu [14].

Definition 1. We call random arrays $\left\{X_{i j}, i=1, \ldots, k, j \geq 1\right\}$

(1) Extended Negatively Lower Orthant Dependent (ENLOD) if there exists some constant $M>0$ such that for each $n=1,2, \ldots,\left\{i_{1}, \ldots, i_{n}\right\} \subset\{1, \ldots, k\}$, $\left\{j_{1}, \ldots, j_{n}\right\} \subset\{1,2, \ldots\}$, and all $x_{1}, \ldots, x_{n}$,

$$
P\left(X_{i_{1} j_{1}} \leq x_{1}, \ldots, X_{i_{n} j_{n}} \leq x_{n}\right) \leq M \prod_{k=1}^{n} P\left(X_{i_{k} j_{k}} \leq x_{k}\right)
$$

(2) Extended Negatively Upper Orthant Dependent (ENUOD) if there exists some constant $M>0$ such that for each $n=1,2, \ldots,\left\{i_{1}, \ldots, i_{n}\right\} \subset\{1, \ldots, k\}$, $\left\{j_{1}, \ldots, j_{n}\right\} \subset\{1,2, \ldots\}$, and all $x_{1}, \ldots, x_{n}$,

$$
P\left(X_{i_{1} j_{1}}>x_{1}, \ldots, X_{i_{n} j_{n}}>x_{n}\right) \leq M \prod_{k=1}^{n} P\left(X_{i_{k} j_{k}}>x_{k}\right) ;
$$

(3) Extended Negatively Orthant Dependent (ENOD) if both (3) and (4) hold for each $n=1,2, \ldots$, $\left\{i_{1}, \ldots, i_{n}\right\} \subset\{1, \ldots, k\},\left\{j_{1}, \ldots, j_{n}\right\} \subset\{1,2, \ldots\}$, and all $x_{1}, \ldots, x_{n}$.

Definition 2. We call random arrays $\left\{X_{i j}, i=1, \ldots, k, j \geq 1\right\}$

(1) Linearly Extended Negatively Lower Orthant Dependent (LENLOD) if for each $m=1,2, \ldots$, any finite disjoint subsets $A_{1}, A_{2}, \ldots, A_{m}$ of $\{1, \ldots, k\} \times$ $\{1,2, \ldots\}$, and positive $r_{i j}{ }^{\prime} \mathrm{s}$,

$$
\begin{gathered}
\sum_{(i, j) \in A_{1}} r_{i j} X_{i j}, \quad \sum_{(i, j) \in A_{2}} r_{i j} X_{i j}, \ldots, \\
\sum_{(i, j) \in A_{m}} r_{i j} X_{i j} \text { are ENLOD; }
\end{gathered}
$$

(2) Linearly Extended Negatively Upper Orthant Dependent (LENUOD) if for each $m=1,2, \ldots$, any finite disjoint subsets $A_{1}, A_{2}, \ldots, A_{m}$ of $\{1, \ldots, k\} \times$ $\{1,2, \ldots\}$, and positive $r_{i j}$ 's,

$$
\begin{gathered}
\sum_{(i, j) \in A_{1}} r_{i j} X_{i j}, \quad \sum_{(i, j) \in A_{2}} r_{i j} X_{i j}, \ldots, \\
\sum_{(i, j) \in A_{m}} r_{i j} X_{i j} \text { are ENUOD; }
\end{gathered}
$$

(3) Linearly Extended Negatively Orthant Dependent (LENOD) if both (5) and (6) hold for each $m=$ $1,2, \ldots$, any finite disjoint subsets $A_{1}, A_{2}, \ldots, A_{m}$ of $\{1, \ldots, k\} \times\{1,2, \ldots\}$ and positive $r_{i j}{ }^{\prime}$ s.

Remark 3. The idea of Definition 2 is due to Newman [27] who first put forward the concept of LNQD (linearly negative quadrant dependent) when studying the central limit theorem. Other related dependence structures are called NQD and NA. See Joag-Dev and Proschan [28] for more details. It is well known that NA implies LNQD and LNQD is slightly stronger than NQD. In the consideration of the need of studying multivariate random variables, we introduce the concept of LENOD random arrays in this paper. By definitions, one can also easily check that NA random arrays must be LENOD ones and LENOD implies ENQD. Moreover, LENOD is more comprehensive than NA in that it can reflect not only a negative association structure but also a positive one to some extent.

Remark 4. It is worth to mention that there maybe exist some flaws in the definition of extended negatively associated (ENA) structure of S. J. Wang and W. S. Wang [21]. In fact, S. J. Wang and W. S. Wang [21] stated that the relationship

$$
\operatorname{Cov}\left(f\left(X_{i j} ;(i, j) \in A\right), g\left(X_{i j} ;(i, j) \in B\right)\right) \leq M
$$

holds for fixed $M>0$ and any pair of coordinatewise increasing functions $f, g$. Considering the case that $\operatorname{Cov}\left(f\left(X_{i j} ;(i, j) \in A\right), g\left(X_{i j} ;(i, j) \in B\right)\right)>0$, inequality (7) can not hold because

$$
\lim _{c \rightarrow \infty} \operatorname{Cov}\left(c f\left(X_{i j} ;(i, j) \in A\right), c g\left(X_{i j} ;(i, j) \in B\right)\right)=\infty .
$$

It might be questionable. Therefore, in this paper, we redefine a new dependence structure called LENOD for random arrays to avoid this problem. Fortunately, under this new dependence structure, the main results still hold and extend some related existing ones. 
The rest of this paper is organized as follows. Section 2 gives some preliminaries. Precise large deviations for nonrandom sums and random sums with dominated variation in dependent multi-risk models are presented in Sections 3 and 4.

\section{Preliminaries}

For convenience, hereafter, for two positive infinitesimals $f(\cdot)$ and $g(\cdot)$ satisfying

$$
a \leq \liminf \frac{f(\cdot)}{g(\cdot)} \leq \limsup \frac{f(\cdot)}{g(\cdot)} \leq b,
$$

we write $f(\cdot)=O(g(\cdot))$ if $b<\infty ; f(\cdot)=o(g(\cdot))$ if $b=0$. For two positive bivariate functions $f(t, x)$ and $g(t, x)$, we say $f(t, x) \sim g(t, x)$ holds as $t \rightarrow \infty$ uniformly for all $x \in$ $\mathscr{D}(t) \neq \phi$ in the sense that $\lim _{t \rightarrow \infty} \sup _{x \in \mathscr{D}(t)} \mid(f(t, x) / g(t, x))-$ $1 \mid=0$.

For simplicity, for any positive integer sequence $\left\{n_{i}, i=\right.$ $1, \ldots, k\}$, we use the following notations:

$$
\begin{gathered}
S_{n}=\sum_{k=1}^{n} X_{k}, \quad S_{n_{i}}=\sum_{j=1}^{n_{i}} X_{i j}, \quad S\left(k ; n_{1}, \ldots, n_{k}\right)=\sum_{i=1}^{k} \sum_{j=1}^{n_{i}} X_{i j}, \\
S_{N(t)}=\sum_{k=1}^{N(t)} X_{k}, \quad S_{N_{i}(t)}=\sum_{k=1}^{N_{i}(t)} X_{i j}, \quad i=1, \ldots, k .
\end{gathered}
$$

2.1. Heavy-Tailed Distributions. In risk theory, heavy-tailed distribution functions are often used to model large claims. They play a key role in some fields such as insurance, financial mathematics, and queueing theory. We say that a nonnegative random variable $X$ (or its distribution function $F$ ) is heavytailed if it has no finite exponential moments. For details, we refer the reader to Embrechts, Klüppelberg, and Mikosch [8], among others. For convenience of use, we recall some important subclasses of heavy-tailed distributions. A quite large subclass is called long-tailed distribution class denoted by $\mathscr{L}$. A distribution function $F$ is said to belong to $\mathscr{L}$ if for all $y \in(-\infty,+\infty)$

$$
\lim _{x \rightarrow \infty} \frac{\bar{F}(x+y)}{\bar{F}(x)}=1 .
$$

Another important subclass is called the class of random variables with dominatedly varying tails (or the class of distribution functions with dominated variation) denoted by $\mathscr{D}$. We say that a distribution function $F$ is in $\mathscr{D}$ if for any $0<y<1$ (or equivalently for some $0<y<1$ )

$$
\limsup _{x \rightarrow \infty} \frac{\bar{F}(x y)}{\bar{F}(x)}<\infty .
$$

Furthermore, for any $y>1$, set $\bar{F}_{*}(y)=$ $\lim _{\inf _{x \rightarrow \infty}}(\bar{F}(x y) / \bar{F}(x))$ and then define

$$
L_{F}=\lim _{y \downarrow 1} \bar{F}_{*}(y), \quad J_{F}^{+}:=\inf \left\{-\frac{\log \bar{F}_{*}(y)}{\log y}, y>1\right\} .
$$

In the terminology of Tang and Tsitsiashvili [29], $J_{F}^{+}$is called the (upper) Matuszewska index of $F$. The following proposition is well known.

Proposition 5. Consider $F \in \mathscr{D} \Leftrightarrow \bar{F}_{*}(y)>0$ for all $y>$ $1 \Leftrightarrow \bar{F}_{*}(y)>0$ for some $y>1 \Leftrightarrow L_{F}>0 \Leftrightarrow J_{F}^{+}<\infty$.

Some other subclasses are as follows. Denote by $\mathscr{C}=\{F$ : $\left.L_{F}=1\right\}$ the class of random variables with consistently varying tails (or distribution functions with consistent variation). For some $0<\alpha \leq \beta<\infty$, denote by $\operatorname{ERV}(-\alpha,-\beta)=\{F$ : $y^{-\beta} \leq \bar{F}_{*}(y) \leq \bar{F}^{*}(y) \leq y^{-\alpha}$ for all $\left.y>1\right\}$ the extended regularly varying class. Particularly, if $\alpha=\beta$, it reduces to the regularly varying class, denoted by $\mathscr{R}_{-\alpha}$. For the heavy-tailed distribution subclasses mentioned above, it is well known that the following inclusions hold:

$$
\mathscr{R}_{-\alpha} \subset \operatorname{ERV}(-\alpha,-\beta) \subset \mathscr{C} \subset \mathscr{D} \cap \mathscr{L} \subset \mathscr{D} \text {. }
$$

Finally, we define an important quantity of heavy-tailed distribution function needed in the main results.

Definition 6. For any heavy-tailed distribution $F$ and $y \geq 0$, define

$$
\rho_{F}(y)=\liminf _{x \rightarrow \infty} \frac{\bar{F}(x+y)}{\bar{F}(x)} .
$$

Remark 7. Obviously, $\rho_{F}(0)=1$. Furthermore, if $F \in \mathscr{L}$, then $\rho_{F}(y) \equiv 1$ for any $y>0$ by the definition of $\mathscr{L}$.

2.2. Some Lemmas. In this sequel, we will give some lemmas needed in the proofs of the main results. Lemmas 1 and 2 are due to S. J. Wang and W. S. Wang [21] (Lemmas 2.3 and 2.5). To state the results, we should introduce two assumptions added on the process $\{N(t), t \geq 0\}$, which also appeared in Chen et al. [15] and S. J. Wang and W. S. Wang [21].

Assumption A. For any $\delta>0$ and some $p>J_{F}^{+}$, as $t \rightarrow \infty$,

$$
\operatorname{EN}^{p}(t) \mathbf{1}_{(N(t)>(1+\delta) \lambda(t))}=O(\lambda(t)) .
$$

Assumption B. For all $0<\delta<1$, as $t \rightarrow \infty$,

$$
P(N(t) \leq(1-\delta) \lambda(t))=o(\lambda(t) \bar{F}(\lambda(t))) .
$$

Lemma 1. Let $\left\{X_{k}, k=1,2, \ldots\right\}$ be ENOD random variables with common distribution $F \in \mathscr{D}$ and finite mean $\mu$, satisfying $E\left(X_{1}^{-}\right)^{r}<\infty$ for some $r>1$ and

$$
F(-x)=o(\bar{F}(x)), \quad x \longrightarrow \infty ;
$$

then, for any $\gamma>0$, as $n \rightarrow \infty$, the relation

$$
P\left(S_{n}-n \mu \leq-x\right)=o(n \bar{F}(x))
$$

holds uniformly for $x \geq \gamma n$.

Lemma 2. Let $\left\{X_{k}, k=1,2, \ldots\right\}$ be a sequence of ENOD random variables with common distribution function $F \in \mathscr{D}$ 
and finite mean $\mu$, satisfying (18), and let $\{N(t), t \geq 0\}$ be a nonnegative integer-valued counting process independent of $\left\{X_{k}, k=1,2, \ldots\right\}$, satisfying Assumptions $A$ and $B$; then, for any $\gamma>0$, as $t \rightarrow \infty$, the relation

$$
P\left(S_{N(t)}-\mu \lambda(t) \leq-x\right)=o(\lambda(t) \bar{F}(x))
$$

holds uniformly for $x \geq \gamma \lambda(t)$.

Lemmas 3-5 play important roles in the proofs of main results and have their own interests. Lemma 3 describes an important property of the quantity $\rho_{F}$ defined in Definition 6. Lemmas 4 and 5 present precise large deviations for nonrandom and random sums of random variables with dominated variation in single-risk models, respectively.

Lemma 3. Let any distribution $F \in \mathscr{D}$, and, for any fixed real number $z$, denote the distribution $F(\cdot+z)$ by $F_{+z}(\cdot)$. Then,

(i) $L_{F} \leq L_{F_{+z}} \leq \rho_{F}^{-1}(z) L_{F}$ when $z \geq 0$;

(ii) $\rho_{F}(-z) L_{F} \leq L_{F_{+z}} \leq L_{F}$ when $z<0$.

Proof. (i) By the definition of $\mathscr{D}$, one can easily check that $F_{+z} \in \mathscr{D}$.

When $z \geq 0$, we get

$$
\begin{aligned}
L_{F_{+z}} & =\lim _{y \downarrow 1} \liminf _{x \rightarrow \infty} \frac{\bar{F}(x y+z)}{\bar{F}(x+z)} \\
& \geq \lim _{y \downarrow 1} \liminf _{x \rightarrow \infty} \frac{\bar{F}((x+z) y)}{\bar{F}(x+z)}=L_{F} .
\end{aligned}
$$

Conversely, for any $0<\varepsilon<1$ and large enough $x$, noticing that $F \in \mathscr{D}$, we have

$$
\begin{aligned}
L_{F_{+z}} & \leq \lim _{y \downarrow 1} \liminf _{x \rightarrow \infty} \frac{\bar{F}(x y)}{\bar{F}(x+z)} \\
& \leq \frac{\lim _{y \downarrow 1} \liminf _{x \rightarrow \infty}(\bar{F}(x y) / \bar{F}(x))}{\liminf \inf _{x \rightarrow \infty}(\bar{F}(x+z) / \bar{F}(x))}=\rho_{F}^{-1}(z) L_{F} .
\end{aligned}
$$

(ii) For any $y \geq 0$ and fixed real number $z$, by Definition 6 , one can easily obtain $\rho_{F_{+z}}(y)=\rho_{F}(y)$. Thus, when $z<0$, (21) and (22) imply $L_{F} \geq L_{F_{+z}}$ and $L_{F} \leq \rho_{F_{+z}}^{-1}(-z) L_{F_{+z}}=$ $\rho_{F}^{-1}(-z) L_{F_{+z}}$. It ends the proof of Lemma 3.

Remark 8. It is interesting that Lemma 3 indicates that the quantity $L_{F_{+z}}$ maybe will not be equal to $L_{F}$ when we let $F_{+z}(\cdot)=F(\cdot+z)$ for any fixed real number $z$. In essence, it is due to the fact that $\mathscr{D}$ is not a subset of $\mathscr{L}$. Thus, we should be very cautious when dealing with the random variables from the subclass $\mathscr{D}$.

Lemma 4. Let $\left\{X_{k}, k \geq 1\right\}$ be a sequence of ENOD random variables with common distribution $F \in \mathscr{D}$ and finite mean $\mu$, satisfying

$$
\begin{gathered}
F(-x)=o(\bar{F}(x)) \quad \text { as } x \longrightarrow \infty, \\
E\left|X_{1}\right|^{r} \mathbf{1}_{\left\{X_{1} \leq 0\right\}}<\infty \quad \text { for some } r>1 .
\end{gathered}
$$

Then, for any fixed $\gamma>0$,

$$
\begin{aligned}
\rho_{F}(|\mu|) L_{F} & \leq \liminf _{n \rightarrow \infty} \inf _{x \geq \gamma n} \frac{P\left(S_{n}-n \mu>x\right)}{n \bar{F}(x)} \\
& \leq \limsup _{n \rightarrow \infty} \sup _{x \geq \gamma n} \frac{P\left(S_{n}-n \mu>x\right)}{n \bar{F}(x)} \leq M_{F, \mu} L_{F}^{-1},
\end{aligned}
$$

where $M_{F, \mu}:=\mathbf{1}_{\{\mu \geq 0\}}+\rho_{F}^{-2}(|\mu|) \mathbf{1}_{\{\mu<0\}}$.

Proof. If $\mu=0$, one can mimic the proof of Theorem 1.1 of Liu [14] to prove (24). Also one can get the relation (24) directly from Corollaries 1 and 2 of Wang et al. [23]. Therefore, we omit the process here.

For the case of $\mu \neq 0$, let $X_{i}^{\prime}=X_{i}-\mu, i=1,2, \ldots$, and note that $X_{i}^{\prime}$ is distributed by $F_{+\mu}$ (see the notation in Lemma 3 ). Therefore, if $\mu<0$, by the fact that $F \in \mathscr{D}$ and Lemma 3, it yields that

$$
\begin{aligned}
& \liminf _{n \rightarrow \infty} \inf _{x \geq \gamma n} \frac{P\left(S_{n}-n \mu>x\right)}{n \bar{F}(x)} \\
& \geq \liminf _{n \rightarrow \infty} \inf _{x \geq \gamma n} \frac{P\left(S_{n}-n \mu>x\right)}{n \bar{F}_{+\mu}(x)} \liminf _{x \rightarrow \infty} \frac{\bar{F}(x+\mu)}{\bar{F}(x)} \\
& \geq L_{F_{+\mu}} \geq \rho_{F}(|\mu|) L_{F}, \\
& \limsup _{n \rightarrow \infty} \sup _{x \geq \gamma n} \frac{P\left(S_{n}-n \mu>x\right)}{n \bar{F}(x)} \\
& \leq \limsup _{n \rightarrow \infty} \sup _{x \geq \gamma n} \frac{P\left(S_{n}-n \mu>x\right)}{n \bar{F}_{+\mu}(x)} \limsup _{x \rightarrow \infty} \frac{\bar{F}(x+\mu)}{\bar{F}(x)} \\
& \leq L_{F_{+\mu}}^{-1}\left(\liminf _{x \rightarrow \infty} \frac{\bar{F}(x)}{\bar{F}(x+\mu)}\right)^{-1} \leq \rho_{F}^{-2}(|\mu|) L_{F}^{-1} .
\end{aligned}
$$

Combining (25) and (26), we get (24). Similarly as above, one can also check that the relation (24) holds if $\mu>0$ and this ends the proof of Lemma 4.

Lemma 5. Let $\left\{X_{k}, k \geq 1\right\}$ be a sequence of ENOD random variables with common distribution $F \in \mathscr{D}$ and finite mean $\mu$, satisfying (23), and let $\{N(t), t \geq 0\}$ be a nonnegative integervalued counting process independent of $\left\{X_{k}, k \geq 1\right\}$. Then, for any fixed $\gamma>0$, the relation

$$
\begin{aligned}
\rho_{F}(|\mu|) L_{F}^{2} & \leq \liminf _{t \rightarrow \infty} \inf _{x \geq \gamma \lambda(t)} \frac{P\left(S_{N(t)}-\mu \lambda(t)>x\right)}{\lambda(t) \bar{F}(x)} \\
& \leq \limsup _{t \rightarrow \infty} \sup _{x \geq \gamma \lambda(t)} \frac{P\left(S_{N(t)}-\mu \lambda(t)>x\right)}{\lambda(t) \bar{F}(x)} \leq M_{F, \mu} L_{F}^{-2}
\end{aligned}
$$

holds under one of the following two conditions (where $M_{F, \mu}$ is defined in Lemma 4):

(i) when $\mu \geq 0$, Assumption A holds;

(ii) when $\mu<0$, Assumption $B$ holds. 
To prove Lemma 5, one can mimic the proof of Theorem 3.1 of S. Wang and X. Wang, [30] by replacing $\mathscr{C}$ by $\mathscr{D}$ and using Lemma 3. For simplicity, we also omit it here.

\section{Large Deviations for Nonrandom Sums}

In this section, we will give precise large deviations for nonrandom sums with dominated variation in multi-risk models under LENOD structures. Hereafter, $M$ always represents a finite and positive constant whose value may vary in different places.

Theorem 9. Let $\left\{X_{i j}, j \geq 1\right\}_{i=1}^{k}$ be LENOD random arrays. For all $i=1, \ldots, k,\left\{X_{i j}, j \geq 1\right\}$ have common distribution function $F_{i}(x)$ and finite expectation $\mu_{i}$, satisfying

$$
F_{i}(-x)=o\left(\bar{F}_{i}(x)\right), \quad x \longrightarrow \infty .
$$

If $E\left|X_{i j}\right|^{r} 1_{\left\{X_{i j} \leq 0\right\}}<\infty$ for some $r>1$ and $F_{i} \in \mathscr{D}$ for all $i=$ $1, \ldots, k$, then, for any fixed $\gamma>0$, we have, for the lower bound,

$$
\liminf _{n_{1}, \ldots, n_{k} \rightarrow \infty} \inf _{x \geq \Delta(k)} \frac{P\left(S\left(k ; n_{1}, \ldots, n_{k}\right)-\sum_{i=1}^{k} n_{i} \mu_{i}>x\right)}{\sum_{i=1}^{k} \rho_{F_{i}}\left(\left|\mu_{i}\right|\right) L_{F_{i}}^{k} n_{i} \bar{F}_{i}(x)} \geq 1,
$$

and, for the upper bound,

$$
\limsup _{n_{1}, \ldots, n_{k} \rightarrow \infty} \sup _{x \geq \Delta(k)} \frac{P\left(S\left(k ; n_{1}, \ldots, n_{k}\right)-\sum_{i=1}^{k} n_{i} \mu_{i}>x\right)}{\sum_{i=1}^{k} M_{F_{i}, \mu_{i}} L_{F_{i}}^{-k} n_{i} \bar{F}_{i}(x)} \leq 1,
$$

where $\Delta(k):=\max \left\{\gamma n_{i}, i=1, \ldots, k\right\}$ and $M_{F_{i}, \mu_{i}}:=\mathbf{1}_{\left\{\mu_{i} \geq 0\right\}}+$ $\rho_{F_{i}}^{-2}\left(\left|\mu_{i}\right|\right) \mathbf{1}_{\left\{\mu_{i}<0\right\}}, i=1,2, \ldots, k$.

Remark 10. In Theorem 9, if we assume $F_{i} \in \mathscr{D} \cap \mathscr{L}$, then (29) and (30) hold with $\rho_{F_{i}}\left(\left|\mu_{i}\right|\right)=M_{F_{i}, \mu_{i}} \equiv 1$ for all $i=1,2, \ldots, k$. Particularly, if $F_{i} \in \mathscr{C}$ for all $i=1, \ldots, k$, then $L_{F_{i}}=1$. Hence, Theorem 9 reduces to Theorem 3.1 of S. J. Wang and W. S. Wang [21]. Moreover, if we also assume $\left\{X_{i j}, j \geq 1\right\}_{i=1}^{k}$ are nonnegative independent random arrays, one can easily see the condition (28) naturally holds. Therefore, (29) and (30) constitute the results of Theorem 3.1 of S. Wang and W. Wang [17].

Remark 11. If we suppose $k=1$ or all $F_{i}(x)(i=1, \ldots, k)$ are the same distribution functions, then Theorem 9 implies Lemma 4. It means that Theorem 9 extends the result of Lemma 4 to multi-risk models. Particularly, if we also assume $F_{1} \in \mathscr{C}$, then Theorem 9 reduces to Theorem 2.1 of Liu [14].

Proof. As usual, we use induction to prove Theorem 9. For the case of $k=2$, we first show that

$$
\liminf _{n_{1}, n_{2} \rightarrow \infty} \inf _{x \geq \Delta(2)} \frac{P\left(S\left(2 ; n_{1}, n_{2}\right)-\sum_{i=1}^{2} n_{i} \mu_{i}>x\right)}{\rho_{F_{1}}\left(\left|\mu_{1}\right|\right) L_{F_{1}}^{2} n_{1} \bar{F}_{1}(x)+\rho_{F_{2}}\left(\left|\mu_{2}\right|\right) L_{F_{2}}^{2} n_{2} \bar{F}_{2}(x)}
$$

$\geq 1$.
By using the same decomposition method of S. J. Wang and W. S. Wang [21], for any $0<\varepsilon<1$ and any $x>0$, it holds that

$$
\begin{aligned}
P( & \left.S\left(2 ; n_{1}, n_{2}\right)-n_{1} \mu_{1}-n_{2} \mu_{2}>x\right) \\
\geq & P\left(S_{n_{1}}-n_{1} \mu_{1}>(1+\varepsilon) x, S_{n_{2}}-n_{2} \mu_{2}>-\varepsilon x\right) \\
& +P\left(S_{n_{2}}-n_{2} \mu_{2}>(1+\varepsilon) x, S_{n_{1}}-n_{1} \mu_{1}>-\varepsilon x\right) \\
& \quad-P\left(S_{n_{1}}-n_{1} \mu_{1}>(1+\varepsilon) x, S_{n_{2}}-n_{2} \mu_{2}>(1+\varepsilon) x\right) \\
:= & K_{1}+K_{2}-K_{3} .
\end{aligned}
$$

For $K_{1}$ and $K_{2}$, by the same argument of relation (3.7) of S. J. Wang and W. S. Wang [21], and Lemmas 1 and 4, one can easily obtain, for any sufficiently small $\delta>0$, that there exist sufficiently large $n_{1}, n_{2}$ such that, uniformly for $x \geq \Delta(2)$,

$$
\begin{aligned}
K_{1} \geq & (1-\delta) \rho_{F_{1}}\left(\left|\mu_{1}\right|\right) L_{F_{1}} n_{1} \bar{F}_{1}((1+\varepsilon) x) \\
& +o\left(\rho_{F_{2}}\left(\left|\mu_{2}\right|\right) L_{F_{2}}^{2} n_{2} \bar{F}_{2}(x)\right) \\
K_{2} \geq & (1-\delta) \rho_{F_{2}}\left(\left|\mu_{2}\right|\right) L_{F_{2}} n_{2} \bar{F}_{2}((1+\varepsilon) x) \\
& +o\left(\rho_{F_{1}}\left(\left|\mu_{1}\right|\right) L_{F_{1}}^{2} n_{1} \bar{F}_{1}(x)\right) .
\end{aligned}
$$

Next we turn to estimate $K_{3}$. Noticing that random arrays $\left\{X_{i j}, i=1,2, \ldots, k, j \geq 1\right\}$ are LENOD, again by Lemma 4 , for sufficiently large $n_{1}, n_{2}$ and uniformly for $x \geq \Delta(2)$, we arrive at

$$
\begin{aligned}
K_{3} \leq & M(1+\delta)^{2} M_{F_{1}, \mu_{1}} L_{F_{1}}^{-1} n_{1} \bar{F}_{1}((1+\varepsilon) x) \\
& \times M_{F_{2}, \mu_{2}} L_{F_{2}}^{-1} n_{2} \bar{F}_{2}((1+\varepsilon) x) \\
\leq & M(1+\delta)^{2} M_{F_{1}, \mu_{1}} L_{F_{1}}^{-1} M_{F_{2}, \mu_{2}} L_{F_{2}}^{-1} n_{1} \bar{F}_{1}(x) n_{2} \bar{F}_{2}(x) \\
= & o\left(\rho_{F_{1}}\left(\left|\mu_{1}\right|\right) L_{F_{1}}^{2} n_{1} \bar{F}_{1}(x)+\rho_{F_{2}}\left(\left|\mu_{2}\right|\right) L_{F_{2}}^{2} n_{2} \bar{F}_{2}(x)\right),
\end{aligned}
$$

where in the last step we use the fact that $\lim _{n_{i} \rightarrow \infty} \sup _{x \geq \gamma n_{i}} n_{i} \bar{F}_{i}(x)=0, i=1,2$. Furthermore, note that $\lim _{\varepsilon \downarrow 0} \liminf \operatorname{in}_{x \rightarrow \infty}\left(\bar{F}_{i}((1+\varepsilon) x) / \bar{F}_{i}(x)\right)=L_{F_{i}}$ for $i=1,2$; hence, for sufficiently small $\varepsilon>0$ and sufficiently large $x$,

$$
\bar{F}_{i}((1+\varepsilon) x) \geq\left(L_{F_{i}}-\delta\right) \bar{F}_{i}(x), \quad i=1,2 .
$$

Combining (32)-(36), it holds that the left-hand side of (32) is bounded from below by

$$
\begin{aligned}
& (1-\delta)\left[\rho_{F_{1}}\left(\left|\mu_{1}\right|\right) L_{F_{1}}\left(L_{F_{1}}-\delta\right) n_{1} \bar{F}_{1}(x)\right. \\
& \left.\quad+\rho_{F_{2}}\left(\left|\mu_{2}\right|\right) L_{F_{2}}\left(L_{F_{2}}-\delta\right) n_{2} \bar{F}_{2}(x)\right] \\
& +o\left(\rho_{F_{1}}\left(\left|\mu_{1}\right|\right) L_{F_{1}}^{2} n_{1} \bar{F}_{1}(x)+\rho_{F_{2}}\left(\left|\mu_{2}\right|\right) L_{F_{2}}^{2} n_{2} \bar{F}_{2}(x)\right) .
\end{aligned}
$$


Therefore, (31) can be derived directly from above by the arbitrariness of $\delta$.

Next we show that

$$
\lim _{n_{1}, n_{2} \rightarrow \infty} \sup _{x \geq \Delta(2)} \frac{P\left(S\left(2 ; n_{1}, n_{2}\right)-n_{1} \mu_{1}-n_{2} \mu_{2}>x\right)}{M_{F_{1}, \mu_{1}} L_{F_{1}}^{-2} n_{1} \bar{F}_{1}(x)+M_{F_{2}, \mu_{2}} L_{F_{2}}^{-2} n_{2} \bar{F}_{2}(x)} \leq 1 .
$$

For any $\varepsilon \in(0,1 / 2)$ and $x>0$, Lemma 4 yields that, for any $0<\delta<1$, sufficiently large $n_{1}, n_{2}$, and uniformly for $x \geq \Delta(2)$,

$$
\begin{aligned}
& P\left(S\left(2 ; n_{1}, n_{2}\right)-n_{1} \mu_{1}-n_{2} \mu_{2}>x\right) \\
& \leq P\left(S_{n_{1}}-n_{1} \mu_{1}>(1-\varepsilon) x\right) \\
& \quad+P\left(S_{n_{2}}-n_{2} \mu_{2}>(1-\varepsilon) x\right) \\
& \quad+M P\left(S_{n_{1}}-n_{1} \mu_{1}>\varepsilon x\right) P\left(S_{n_{2}}-n_{2} \mu_{2}>\varepsilon x\right) \\
& \leq(1+\delta)\left[M_{F_{1}, \mu_{1}} L_{F_{1}}^{-1} n_{1} \bar{F}_{1}((1-\varepsilon) x)\right. \\
& \left.\quad+M_{F_{2}, \mu_{2}} L_{F_{2}}^{-1} n_{2} \bar{F}_{2}((1-\varepsilon) x)\right] \\
& \quad+M(1+\delta)^{2} M_{F_{1}, \mu_{1}} L_{F_{1}}^{-1} n_{1} \bar{F}_{1}(\varepsilon x) M_{F_{2}, \mu_{2}} L_{F_{2}}^{-1} n_{2} \bar{F}_{2}(\varepsilon x) .
\end{aligned}
$$

Note that

$$
\begin{gathered}
\frac{n_{1} \bar{F}_{1}(\varepsilon x) n_{2} \bar{F}_{2}(\varepsilon x)}{M_{F_{1}, \mu_{1}} L_{F_{1}}^{-2} n_{1} \bar{F}_{1}(x)+M_{F_{2}, \mu_{2}} L_{F_{2}}^{-2} n_{2} \bar{F}_{2}(x)} \\
=\left(M_{F_{1}, \mu_{1}} L_{F_{1}}^{-2} \frac{\bar{F}_{1}(x)}{\bar{F}_{1}(\varepsilon x)} \frac{1}{n_{2} \bar{F}_{2}(\varepsilon x)}\right. \\
\left.+M_{F_{2}, \mu_{2}} L_{F_{2}}^{-2} \frac{\bar{F}_{2}(x)}{\bar{F}_{2}(\varepsilon x)} \frac{1}{n_{1} \bar{F}_{1}(\varepsilon x)}\right)^{-1} .
\end{gathered}
$$

It is easy to check that $\lim _{n_{i} \rightarrow \infty} \sup _{x \geq \gamma n_{i}} n_{i} \bar{F}_{i}(\varepsilon x)=0$, $i=1,2$. Moreover, since $F_{1}, F_{2} \in \mathscr{D}$, we have, for any $0<\varepsilon<1, \liminf _{n_{1} \rightarrow \infty} \inf _{x \geq \gamma n_{1}}\left(\bar{F}_{1}(x) / \bar{F}_{1}(\varepsilon x)\right)>0$ and $\lim \inf _{n_{2} \rightarrow \infty} \inf _{x \geq \gamma n_{2}}\left(\bar{F}_{2}(x) / \bar{F}_{2}(\varepsilon x)\right)>0$. Therefore, we have, as $n_{1}, n_{2} \rightarrow \infty$, uniformly for $x \geq \Delta(2)$,

$$
\begin{aligned}
& n_{1} \bar{F}_{1}(\varepsilon x) n_{2} \bar{F}_{2}(\varepsilon x) \\
& \quad=o\left(M_{F_{1}, \mu_{1}} L_{F_{1}}^{-2} n_{1} \bar{F}_{1}(x)+M_{F_{2}, \mu_{2}} L_{F_{2}}^{-2} n_{2} \bar{F}_{2}(x)\right) .
\end{aligned}
$$

Furthermore, by the definition of $L_{F_{i}}$, for sufficiently small $\varepsilon>0$ and sufficiently large $x$, similarly as (36), it yields that

$$
\bar{F}_{i}((1-\varepsilon) x) \leq\left(L_{F_{i}}^{-1}+\delta\right) \bar{F}_{i}(x), \quad i=1,2 .
$$

Substituting (41) and (42) into (39) and letting $\delta \downarrow 0$, (38) holds and Theorem 9 is proved in the case of $k=2$.
Now suppose (29) holds for $k-1$; for the case of $k$, using the similar argument as (32), it holds that

$$
\begin{aligned}
P(S & \left.\left(k ; n_{1}, \ldots, n_{k}\right)-\sum_{i=1}^{k} n_{i} \mu_{i}>x\right) \\
\geq & P\left(\sum_{i=1}^{k-1} S_{n_{i}}-\sum_{i=1}^{k-1} n_{i} \mu_{i}>(1+\varepsilon) x, S_{n_{k}}-n_{k} \mu_{k}>-\varepsilon x\right) \\
& +P\left(\sum_{i=1}^{k-1} S_{n_{i}}-\sum_{i=1}^{k-1} n_{i} \mu_{i}>-\varepsilon x, S_{n_{k}}-n_{k} \mu_{k}>(1+\varepsilon) x\right) \\
& -M P\left(\sum_{i=1}^{k-1} S_{n_{i}}-\sum_{i=1}^{k-1} n_{i} \mu_{i}>(1+\varepsilon) x\right) \\
\quad & P\left(S_{n_{k}}-n_{k} \mu_{k}>(1+\varepsilon) x\right) \\
:= & I_{1}+I_{2}-M I_{3} .
\end{aligned}
$$

To estimate $I_{1}$ and $I_{2}$, similarly as (33) and (34), by Lemma 1, Lemma 4 , and induction hypothesis, we have, for any $0<\delta<$ 1 , all $i=1, \ldots, k$, sufficiently small $\varepsilon>0$, as $n_{i} \rightarrow \infty$,

$$
\begin{aligned}
I_{1} \geq & (1-\delta) \sum_{i=1}^{k-1} \rho_{F_{i}}\left(\left|\mu_{i}\right|\right) L_{F_{i}}^{k-1}\left(L_{F_{i}}-\delta\right) n_{i} \bar{F}_{i}(x) \\
& +o\left(\rho_{F_{k}}\left(\left|\mu_{k}\right|\right) L_{F_{k}}^{k} n_{k} \bar{F}_{k}(x)\right) \\
I_{2} \geq & (1-\delta) \rho_{F_{k}}\left(\left|\mu_{k}\right|\right) L_{F_{k}}^{k-1}\left(L_{F_{k}-} \delta\right) n_{k} \bar{F}_{k}(x) \\
& +o\left(\sum_{i=1}^{k-1} \rho_{F_{i}}\left(\left|\mu_{i}\right|\right) L_{F_{i}}^{k} n_{i} \bar{F}_{i}(x)\right) .
\end{aligned}
$$

Finally for $I_{3}$, similarly as (35), we arrive at

$$
I_{3}=o\left(\sum_{i=1}^{k} \rho_{F_{i}}\left(\left|\mu_{i}\right|\right) L_{F_{i}}^{k} n_{i} \bar{F}_{i}(x)\right) .
$$

Combining (43)-(45) and letting $\delta \downarrow 0$, we have

$$
\liminf _{n_{1}, \ldots, n_{k} \rightarrow \infty} \inf _{x \geq \Delta(k)} \frac{P\left(S\left(k ; n_{1}, \ldots, n_{k}\right)-\sum_{i=1}^{k} n_{i} \mu_{i}>x\right)}{\sum_{i=1}^{k} \rho_{F_{i}}\left(\left|\mu_{i}\right|\right) L_{F_{i}}^{k} n_{i} \bar{F}_{i}(x)} \geq 1 .
$$


To obtain the reverse inequality, for any $\varepsilon \in(0,1 / 2)$ and $x>$ 0 , by Lemma 4 and induction hypothesis, it holds that, for any $0<\delta<1$, sufficiently small $\varepsilon>0$,

$$
\begin{aligned}
& P\left(S\left(k ; n_{1}, \ldots, n_{k}\right)-\sum_{i=1}^{k} n_{i} \mu_{i}>x\right) \\
& \leq P\left(\sum_{i=1}^{k-1} S_{n_{i}}-\sum_{i=1}^{k-1} n_{i} \mu_{i}>(1-\varepsilon) x\right) \\
& +P\left(S_{n_{k}}-n_{k} \mu_{k}>(1-\varepsilon) x\right) \\
& \quad+M P\left(\sum_{i=1}^{k-1} S_{n_{i}}-\sum_{i=1}^{k-1} n_{i} \mu_{i}>\varepsilon x\right) P\left(S_{n_{k}}-n_{k} \mu_{k}>\varepsilon x\right) \\
& \leq(1+\delta)\left[\sum_{i=1}^{k-1} M_{F_{i}, \mu_{i}} L_{F_{i}}^{-(k-1)} n_{i} \bar{F}_{i}((1-\varepsilon) x)\right. \\
& +o\left(\sum_{i=1}^{k} M_{F_{i}, \mu_{i}} L_{F_{i}}^{-k} n_{i} \bar{F}_{i}(x)\right) \\
& +(1+\delta)^{2} M \sum_{i=1}^{k-1} M_{F_{i}, \mu_{i}} L_{F_{i}}^{-(k-1)} n_{i} \bar{F}_{i}(\varepsilon x) \\
& \left.+\sum_{F_{k}, \mu_{k}} L_{F_{k}}^{-1} n_{k} \bar{F}_{k}((1-\varepsilon) x)\right] \\
& \times M_{F_{k}, \mu_{k}} L_{F_{k}}^{-1} n_{k} \bar{F}_{k}(\varepsilon x) \\
& +M_{F_{i}}^{-(k-1)}\left(L_{F_{i}}^{-1}+\delta\right) n_{i} \bar{F}_{i}(x)
\end{aligned}
$$

Letting $\delta \downarrow 0$, we get

$$
\limsup _{n_{1}, \ldots, n_{k} \rightarrow \infty} \sup _{x \geq \Delta(k)} \frac{P\left(S\left(k ; n_{1}, \ldots, n_{k}\right)-\sum_{i=1}^{k} n_{i} \mu_{i}>x\right)}{\sum_{i=1}^{k} M_{F_{i}, \mu_{i}} L_{F_{i}}^{-k} n_{i} \bar{F}_{i}(x)} \leq 1 .
$$

Therefore, Theorem 9 follows from (46) and (48) immediately.

\section{Large Deviations for Random Sums}

In this section, we will give precise large deviations for random sums with dominated variation in multi-risk models under LENOD structures.

Remark 12. It is worth to mention that Assumption B was firstly introduced by Chen et al. [15]. Furthermore, one can easily see that both Assumptions A and B imply that

$$
\frac{N(t)}{\lambda(t)} \stackrel{P}{\longrightarrow} 1
$$

Theorem 13. Let $\left\{X_{i j}, j \geq 1\right\}_{i=1}^{k}$ be LENOD random arrays satisfying the conditions of Theorem 9, and let $\left\{N_{i}(t)\right\}_{i=1}^{k}$ be independent nonnegative integer-valued process independent of $\left\{X_{i j}, j \geq 1\right\}_{i=1}^{k}$, satisfying Assumptions $A$ and $B$; then, for any fixed $\gamma>0$, we have, for the lower bound,

$$
\liminf _{t \rightarrow \infty} \inf _{x \geq \Gamma(k)} \frac{P\left(S(k ; t)-\sum_{i=1}^{k} \lambda_{i}(t) \mu_{i}>x\right)}{\sum_{i=1}^{k} \rho_{F_{i}}\left(\left|\mu_{i}\right|\right) L_{F_{i}}^{k+1} \lambda_{i}(t) \bar{F}_{i}(x)} \leq 1,
$$

and, for the upper bound,

$$
\limsup _{t \rightarrow \infty} \sup _{x \geq \Gamma(k)} \frac{P\left(S(k ; t)-\sum_{i=1}^{k} \lambda_{i}(t) \mu_{i}>x\right)}{\sum_{i=1}^{k} M_{F_{i}, \mu_{i}} L_{F_{i}}^{-(k+1)} \lambda_{i}(t) \bar{F}_{i}(x)} \leq 1,
$$

where $\Gamma(k):=\max \left\{\gamma \lambda_{i}(t), i=1, \ldots, k\right\}$ and $M_{F_{i}, \mu_{i}}$ is defined in Theorem 9.

Remark 14. In Theorem 13, if we assume $F_{i} \in \mathscr{D} \cap \mathscr{L}$, then (50) and (51) hold with $\rho_{F_{i}}\left(\left|\mu_{i}\right|\right)=M_{F_{i}, \mu_{i}} \equiv 1$ for all $i=1,2, \ldots, k$. Particularly, if $F_{i} \in \mathscr{C}$ for all $i=1, \ldots, k$, then $L_{F_{i}}=1$. Hence, Theorem 13 reduces to Theorem 4.1 of S. J. Wang and W. S. Wang [21]. Moreover, if we also assume $\left\{X_{i j}, j \geq 1\right\}_{i=1}^{k}$ are nonnegative independent random arrays, one can easily see the condition (28) naturally holds. Therefore, (50) and (51) constitute the results of Theorem 4.1 of S. Wang and W. Wang [17].

Remark 15. If we suppose $k=1$ or all $F_{i}(x)(i=1, \ldots, k)$ are the same distribution function, then Theorem 13 implies Lemma 5. It means that Theorem 13 extends the result of Lemma 5 to multi-risk models. Particularly, if we also assume $F_{1} \in \mathscr{C}$, then Theorem 13 indicates Theorem 3.1 of Chen et al. [15] and Theorem 3.2 of S. Wang and X. Wang [30].

Proof. Similarly as the proof of Theorem 9, again by induction, it is sufficient to show that Theorem 13 holds for $k=2$. We first show that

$$
\liminf _{t \rightarrow \infty} \inf _{x \geq \Gamma(2)} \frac{P\left(S(2 ; t)-\lambda_{1}(t) \mu_{1}-\lambda_{2}(t) \mu_{2}>x\right)}{\rho_{F_{1}}\left(\left|\mu_{1}\right|\right) L_{F_{1}}^{3} \lambda_{1}(t) \bar{F}_{1}(x)+\rho_{F_{2}}\left(\left|\mu_{2}\right|\right) L_{F_{2}}^{3} \lambda_{2}(t) \bar{F}_{2}(x)}
$$

$\geq 1$.

The similar argument as (32) yields that, for any $0<\varepsilon<1$ and any $x>0$,

$$
\begin{gathered}
P\left(S(2 ; t)-\lambda_{1}(t) \mu_{1}-\lambda_{2}(t) \mu_{2}>x\right) \\
\geq P\left(S_{N_{1}(t)}-\lambda_{1}(t) \mu_{1}>(1+\varepsilon) x\right. \\
\left.S_{N_{2}(t)}-\lambda_{2}(t) \mu_{2}>-\varepsilon x\right)
\end{gathered}
$$




$$
\begin{gathered}
+P\left(S_{N_{2}(t)}-\lambda_{2}(t) \mu_{2}>(1+\varepsilon) x,\right. \\
\left.S_{N_{1}(t)}-\lambda_{1}(t) \mu_{1}>-\varepsilon x\right) \\
-P\left(S_{N_{1}(t)}-\lambda_{1}(t) \mu_{1}>(1+\varepsilon) x,\right. \\
\left.\quad S_{N_{2}(t)}-\lambda_{2}(t) \mu_{2}>(1+\varepsilon) x\right) \\
:=J_{1}+J_{2}-J_{3} .
\end{gathered}
$$

To deal with $J_{1}$, note that

$$
\begin{aligned}
J_{1} \geq & P\left(S_{N_{1}(t)}-\lambda_{1}(t) \mu_{1}>(1+\varepsilon) x\right) \\
& -P\left(S_{N_{2}(t)}-\lambda_{2}(t) \mu_{2} \leq-\varepsilon x\right) .
\end{aligned}
$$

It follows from Lemmas 2 and 5 that, for any $\delta>0$,

$$
\begin{aligned}
J_{1} \geq & (1-\delta) \rho_{F_{1}}\left(\left|\mu_{1}\right|\right) L_{F_{1}}^{2}\left(L_{F_{1}}-\delta\right) \lambda_{1}(t) \bar{F}_{1}(x) \\
& +o\left(\rho_{F_{2}}\left(\left|\mu_{2}\right|\right) L_{F_{2}}^{3} \lambda_{2}(t) \bar{F}_{2}(x)\right), \\
J_{2} \geq & (1-\delta) \rho_{F_{2}}\left(\left|\mu_{2}\right|\right) L_{F_{2}}^{2}\left(L_{F_{2}}-\delta\right) \lambda_{2}(t) \bar{F}_{2}(x) \\
& +o\left(\rho_{F_{1}}\left(\left|\mu_{1}\right|\right) L_{F_{1}}^{3} \lambda_{1}(t) \bar{F}_{1}(x)\right) .
\end{aligned}
$$

Next we turn to estimate $J_{3}$. Note that $\left\{N_{i}(t)\right\}_{i=1}^{2}$ being independent and $\left\{X_{i j}, j \geq 1\right\}_{i=1}^{2}$ being LENOD, by Lemma 5 , one gets

$$
\begin{aligned}
J_{3} \leq & M P\left(S_{N_{1}(t)}-\lambda_{1}(t) \mu_{1}>(1+\varepsilon) x\right) \\
& \times P\left(S_{N_{2}(t)}-\lambda_{2}(t) \mu_{2}>(1+\varepsilon) x\right) \\
\leq & M(1+\delta)^{2} M_{F_{1}, \mu_{1}} L_{F_{1}}^{-2} \lambda_{1}(t) \bar{F}_{1}(x) M_{F_{2}, \mu_{2}} \\
& \times L_{F_{2}}^{-2} \lambda_{2}(t) \bar{F}_{2}(x) \\
= & o\left(\rho_{F_{1}}\left(\left|\mu_{1}\right|\right) L_{F_{1}}^{3} \lambda_{1}(t) \bar{F}_{1}(x)\right. \\
& \left.\quad+\rho_{F_{2}}\left(\left|\mu_{2}\right|\right) L_{F_{2}}^{3} \lambda_{2}(t) \bar{F}_{2}(x)\right),
\end{aligned}
$$

where in the last step we use the fact that $\lim _{t \rightarrow \infty} \sup _{x \geq \gamma \lambda_{i}(t)} \lambda_{i}(t) \bar{F}_{i}(x)=0, i=1,2$. Therefore, by (53)-(57), for any sufficiently large $t$ and uniformly for $x \geq \Gamma(2)$,

$$
\begin{gathered}
P\left(S(2 ; t)-\lambda_{1}(t) \mu_{1}-\lambda_{2}(t) \mu_{2}>x\right) \\
\geq(1-\delta)\left[\rho_{F_{1}}\left(\left|\mu_{1}\right|\right) L_{F_{1}}^{2}\left(L_{F_{1}}-\delta\right) \lambda_{1}(t) \bar{F}_{1}(x)\right. \\
\left.+\rho_{F_{2}}\left(\left|\mu_{2}\right|\right) L_{F_{2}}^{2}\left(L_{F_{2}}-\delta\right) \lambda_{2}(t) \bar{F}_{2}(x)\right] \\
+o\left(\rho_{F_{1}}\left(\left|\mu_{1}\right|\right) L_{F_{1}}^{3} \lambda_{1}(t) \bar{F}_{1}(x)\right. \\
\left.+\rho_{F_{2}}\left(\left|\mu_{2}\right|\right) L_{F_{2}}^{3} \lambda_{2}(t) \bar{F}_{2}(x)\right) .
\end{gathered}
$$

Letting $\delta \downarrow 0$, (52) derives directly from the inequality above.
Next we show that

$$
\limsup _{t \rightarrow \infty} \sup _{x \geq \Gamma(2)} \frac{P\left(S(2 ; t)-\lambda_{1}(t) \mu_{1}-\lambda_{2}(t) \mu_{2}>x\right)}{M_{F_{1}, \mu_{1}} L_{F_{1}}^{-3} \lambda_{1}(t) \bar{F}_{1}(x)+M_{F_{2}, \mu_{2}} L_{F_{2}}^{-3} \lambda_{2}(t) \bar{F}_{2}(x)}
$$

$\leq 1$

For any $\in(0,1 / 2), x>0,0<\delta<1$, by Lemma 5 , (41), and (42), we arrive at

$$
\begin{aligned}
& P\left(S(2 ; t)-\lambda_{1}(t) \mu_{1}-\lambda_{2}(t) \mu_{2}>x\right) \\
& \leq P\left(S_{N_{1}(t)}-\lambda_{1}(t) \mu_{1}>(1-\varepsilon) x\right) \\
&+P\left(S_{N_{2}(t)}-\lambda_{2}(t) \mu_{2}>(1-\varepsilon) x\right) \\
&+M P\left(S_{N_{1}(t)}-\lambda_{1}(t) \mu_{1}>\varepsilon x\right) \\
& \times P\left(S_{N_{2}(t)}-\lambda_{2}(t) \mu_{2}>\varepsilon x\right) \\
& \leq(1+\delta)\left[M_{F_{1}, \mu_{1}} L_{F_{1}}^{-2} \lambda_{1}(t) \bar{F}_{1}((1-\varepsilon) x)\right. \\
&\left.\quad+M_{F_{2}, \mu_{2}} L_{F_{2}}^{-2} \lambda_{2}(t) \bar{F}_{2}((1-\varepsilon) x)\right] \\
&+(1+\delta)^{2} M_{F_{1}, \mu_{1}} L_{F_{1}}^{-2} \lambda_{1}(t) \bar{F}_{1}(\varepsilon x) M_{F_{2}, \mu_{2}} \\
& \times L_{F_{2}}^{-2} \lambda_{2}(t) \bar{F}_{2}(\varepsilon x) \\
& \leq(1+\delta)\left[M_{F_{1}, \mu_{1}} L_{F_{1}}^{-2}\left(L_{F_{1}}^{-1}+\delta\right) \lambda_{1}(t) \bar{F}_{1}(x)\right. \\
&\left.\quad+M_{F_{2}, \mu_{2}} L_{F_{2}}^{-2}\left(L_{F_{2}}^{-1}+\delta\right) \lambda_{2}(t) \bar{F}_{2}(x)\right] \\
&+o\left(M_{F_{1}, \mu_{1}} L_{F_{1}}^{-3} \lambda_{1}(t) \bar{F}_{1}(x)\right. \\
&\left.+M_{F_{2}, \mu_{2}} L_{F_{2}}^{-3} \lambda_{2}(t) \bar{F}_{2}(x)\right) .
\end{aligned}
$$

Letting $\delta \downarrow 0$, thus we get (59).

Combining (52) and (59), it indicates that Theorem 13 holds for $k=2$. Finally, by induction similar as Theorem 9, the proof of Theorem 13 is now completed.

\section{Conflict of Interests}

The authors declare that there is no conflict of interests regarding the publication of this paper.

\section{Acknowledgments}

The authors would like to thank the academic editor Beong In Yun and the anonymous referees for their constructive and insightful suggestions that greatly improved the paper. This work was partially supported by the National Natural Science Foundation of China (11226207, 11201001), the Natural Science Foundation of Anhui Province (1208085QA03), the Project of Applicative Teaching Demonstration Courses of Anhui University (XJYYXKC09), the Project Sponsored by the Doctoral Scientific Research Foundation of Anhui University, and the Students Science Research Training Program of Anhui University (KYXL2014008). 


\section{References}

[1] A. V. Nagaev, "Integral limit theorems with regard to large deviations when Cramér's condition is not satisfied. I," Theory of Probability and Its Applications, vol. 14, pp. 51-64, 1969.

[2] A. V. Nagaev, "Integral limit theorems with regard to large deviations when Cramér's condition is not satisfied," Theory of Probability and Its Applications, vol. 14, no. 1, pp. 193-208, 1969.

[3] C. C. Heyde, "A contribution to the theory of large deviations for sums of independent random variables," vol. 7, pp. 303-308, 1967.

[4] C. C. Heyde, "On large deviation problems for sums of random variables which are not attracted to the normal law," Annals of Mathematical Statistics, vol. 38, pp. 1575-1578, 1967.

[5] S. V. Nagaev, "Large deviations for sums of independent random variables," in Transactions of the Sixth Prague Conference on Information Theory, Statistical Decision Functions, Random Processes, pp. 657-674, Academia, Prague, Czech Republic, 1973.

[6] S. V. Nagaev, "Large deviations of sums of independent random variables," The Annals of Probability, vol. 7, no. 5, pp. 745-789, 1979.

[7] D. B. H. Cline and T. Hsing, Large Deviation Probabilities for Sums and Maxima of Random Variables with Heavy or Subexponential Tails, Texaxs A\&M University, 1991.

[8] C. Klüppelberg and T. Mikosch, "Large deviations of heavytailed random sums with applications in insurance and finance," Journal of Applied Probability, vol. 34, no. 2, pp. 293-308, 1997.

[9] Q. Tang, C. Su, T. Jiang, and J. Zhang, "Large deviations for heavy-tailed random sums in compound renewal model," Statistics \& Probability Letters, vol. 52, no. 1, pp. 91-100, 2001.

[10] Q. H. Tang, "Insensitivity to negative dependence of the asymptotic behavior of precise large deviations," Electronic Journal of Probability, vol. 11, no. 4, pp. 107-120, 2006.

[11] Y. Liu, "Precise large deviations for negatively associated random variables with consistently varying tails," Statistics \& Probability Letters, vol. 77, no. 2, pp. 181-189, 2007.

[12] Y. Chen and W. Zhang, "Large deviations for random sums of negatively dependent random variables with consistently varying tails," Statistics \& Probability Letters, vol. 77, no. 5, pp. 530-538, 2007.

[13] X. M. Shen and Z. Y. Lin, "Precise large deviations for randomly weighted sums of negatively dependent random variables with consistently varying tails," Statistics and Probability Letters, vol. 78, no. 18, pp. 3222-3229, 2008.

[14] L. Liu, "Precise large deviations for dependent random variables with heavy tails," Statistics \& Probability Letters, vol. 79, no. 9, pp. 1290-1298, 2009.

[15] Y. Q. Chen, K. C. Yuen, and K. W. Ng, "Precise large deviations of random sums in presence of negative dependence and consistent variation," Methodology and Computing in Applied Probability, vol. 13, no. 4, pp. 821-833, 2011.

[16] Y. Q. Chen and K. C. Yuen, "Precise large deviations of aggregate claims in a size-dependent renewal risk model," Insurance: Mathematics \& Economics, vol. 51, no. 2, pp. 457-461, 2012.

[17] S. Wang and W. Wang, "Precise large deviations for sums of random variables with consistently varying tails in multi-risk models," Journal of Applied Probability, vol. 44, no. 4, pp. 889900, 2007.

[18] D. Lu, "Lower and upper bounds of large deviation for sums of subexponential claims in a multi-risk model," Statistics \& Probability Letters, vol. 81, no. 12, pp. 1911-1919, 2011.
[19] D. Lu, "Lower bounds of large deviation for sums of long-tailed claims in a multi-risk model," Statistics \& Probability Letters, vol. 82, no. 7, pp. 1242-1250, 2012.

[20] W. He, D. Cheng, and Y. Wang, "Asymptotic lower bounds of precise large deviations with nonnegative and dependent random variables," Statistics \& Probability Letters, vol. 83, no. 1, pp. 331-338, 2013.

[21] S. J. Wang and W. S. Wang, "Precise large deviations for sums of random variables with consistent variation in dependent multirisk models," Communications in Statistics: Theory and Methods, vol. 42, no. 24, pp. 4444-4459, 2013.

[22] Y. B. Wang, K. Y. Wang, and D. Y. Cheng, "Precise large deviations for sums of negatively associated random variables with common dominatedly varying tails," Acta Mathematica Sinica, vol. 22, no. 6, pp. 1725-1734, 2006.

[23] K. Wang, Y. Yang, and J. Lin, "Precise large deviations for widely orthant dependent random variables with dominatedly varying tails," Frontiers of Mathematics in China, vol. 7, no. 5, pp. 919932, 2012.

[24] Y. Yang, R. Leipus, and J. Šiaulys, "Precise large deviations for compound random sums in the presence of dependence structures," Computers \& Mathematics with Applications, vol. 64, no. 6, pp. 2074-2083, 2012.

[25] Y. Yang and K. Wang, "Precise large deviations for dependent random variables with applications to the compound renewal risk model," The Rocky Mountain Journal of Mathematics, vol. 43, no. 4, pp. 1395-1414, 2013.

[26] Y. Chen and Z. Qu, "Precise large deviations for random sums of END random variables with dominated variation," ISRN Applied Mathematics, vol. 2013, Article ID 936301, 10 pages, 2013.

[27] C. M. Newman, "Asymptotic independence and limit theorems for positively and negatively dependent random variables," in Inequalities in Statistics and Probability, Y. L. Tong, Ed., vol. 5 of IMS Lectures Notes-Monograph Series, pp. 127-140, Institute of Mathematical Statistics, Hayward, Calif, USA, 1984.

[28] K. Joag-Dev and F. Proschan, "Negative association of random variables, with applications," The Annals of Statistics, vol. 11, no. 1, pp. 286-295, 1983.

[29] Q. H. Tang and G. Tsitsiashvili, "Precise estimates for the ruin probability in finite horizon in a discrete-time model with heavy-tailed insurance and financial risks," Stochastic Processes and Their Applications, vol. 108, no. 2, pp. 299-325, 2003.

[30] S. Wang and X. Wang, "Precise large deviations for random sums of END real-valued random variables with consistent variation," Journal of Mathematical Analysis and Applications, vol. 402, no. 2, pp. 660-667, 2013. 


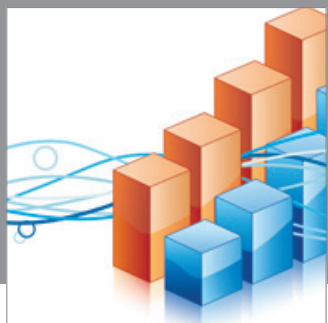

Advances in

Operations Research

mansans

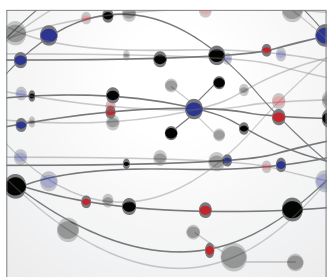

The Scientific World Journal
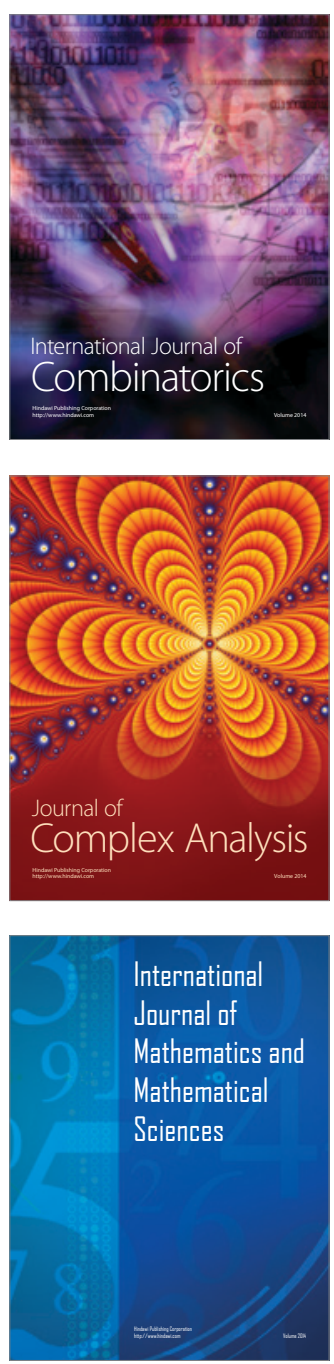
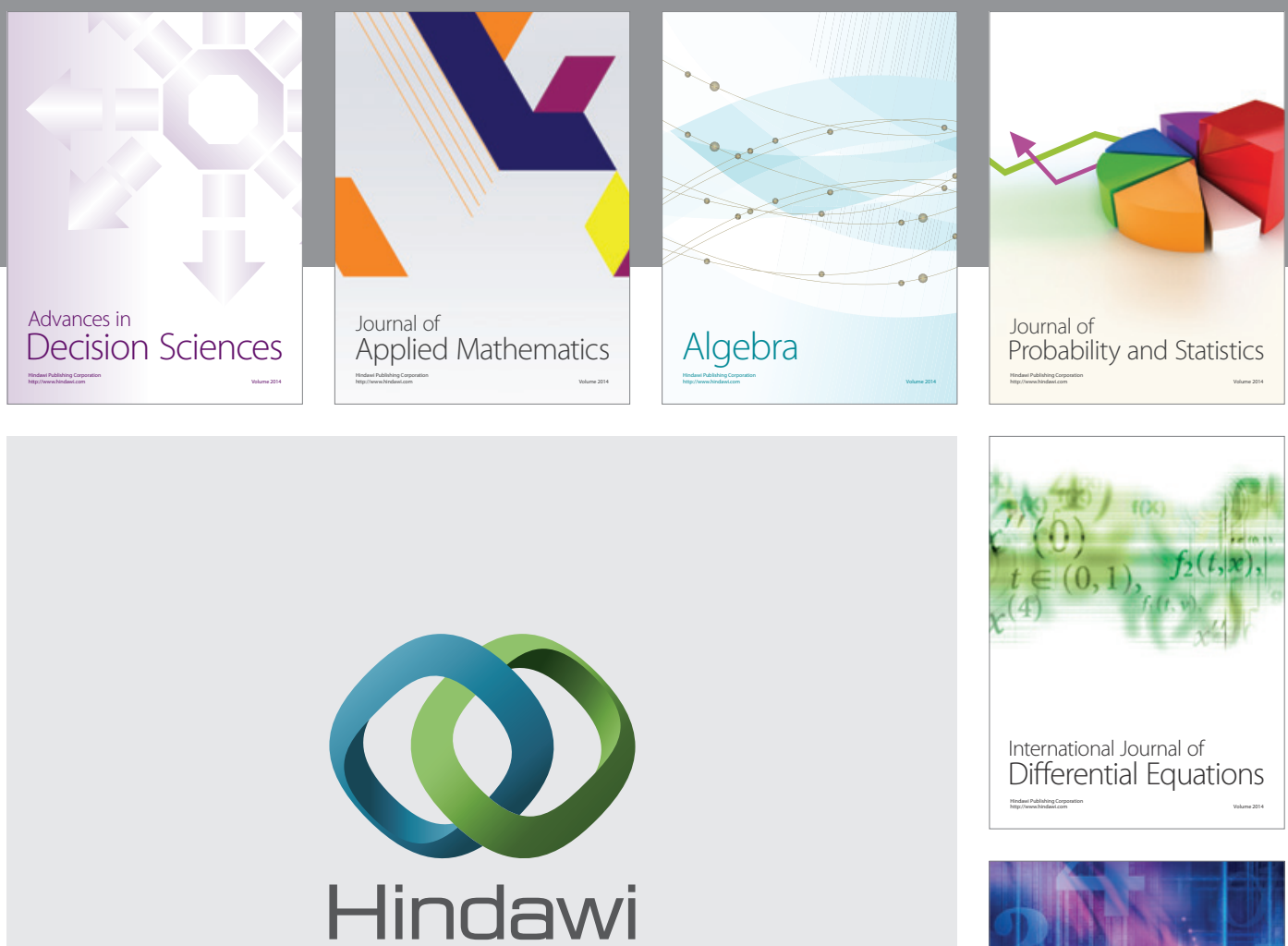

Submit your manuscripts at http://www.hindawi.com
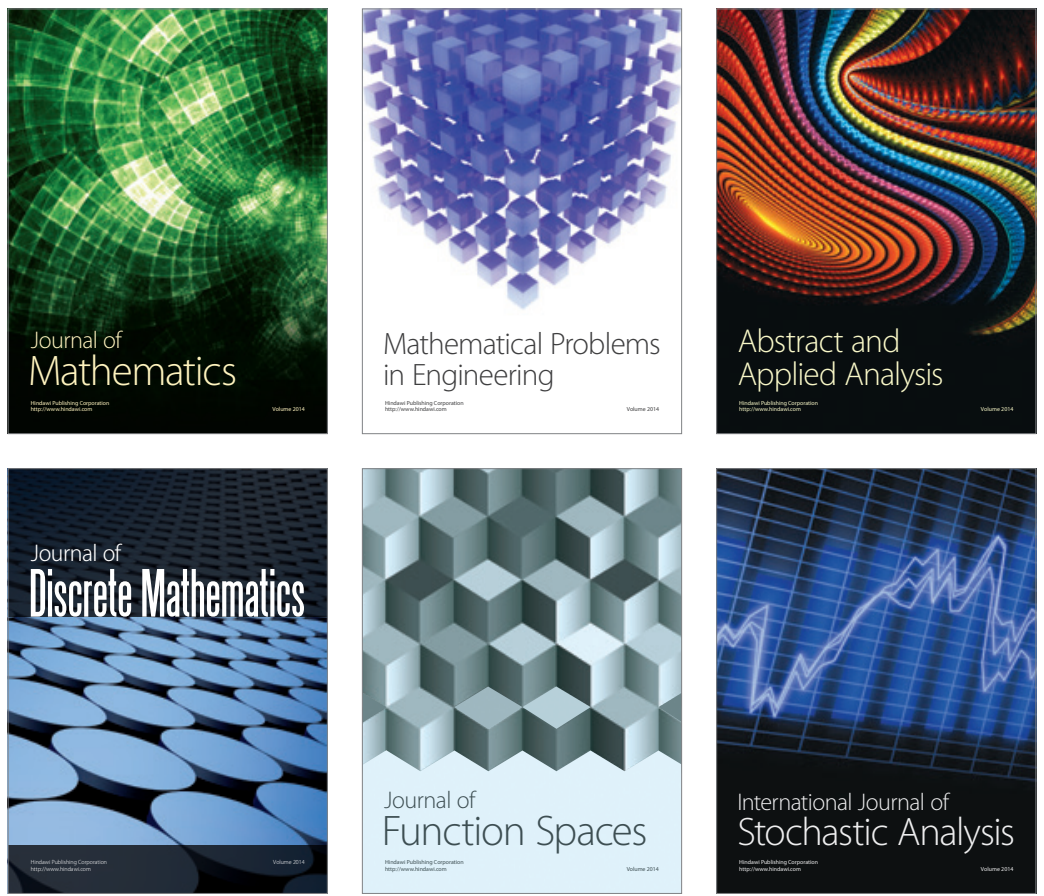

Journal of

Function Spaces

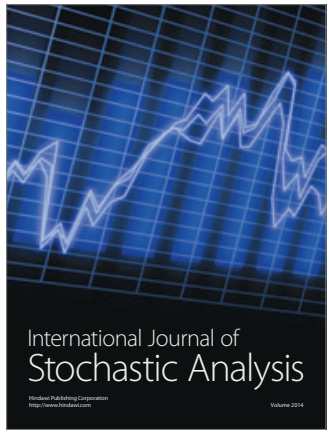

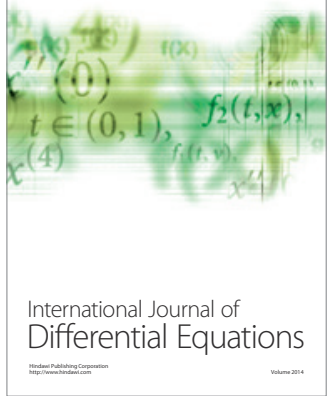
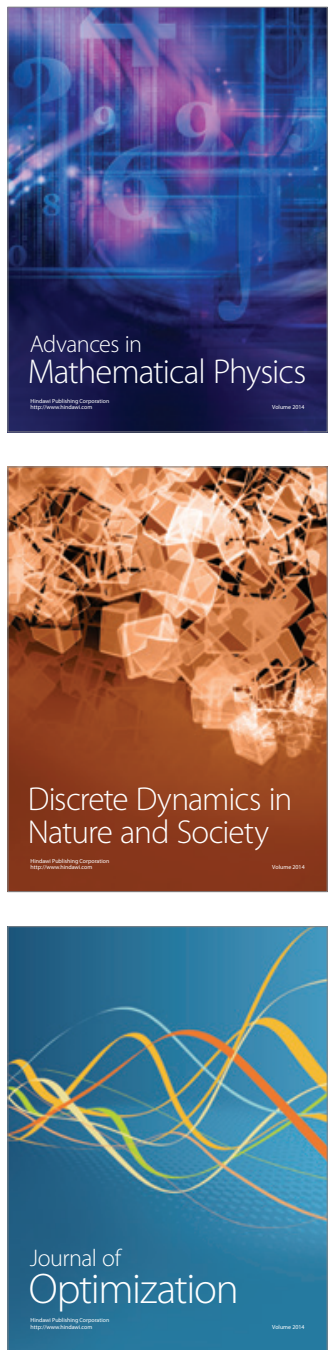